\title{
TROCAS GASOSAS E CONDUTÂNCIA ESTOMÁTICA EM TRÊS ESPÉCIES DE GRAMÍNEAS $\left({ }^{1}\right)$
}

EDUARDO CARUSO MACHADO $\left({ }^{2,3}\right)$ e ANA MARIA MAGALHÃES ANDRADE LAGÔA $\left(^{2,3}\right.$ )

\begin{abstract}
RESUMO
Avaliou-se, sob condiçōes naturais e sem deficiência hídrica, o comportamento diário das taxas de assimilação de $\mathrm{CO}_{2}(A)$ e de transpiração $(E)$, a condutância estomática $(g)$ e a eficiência fotossintética do uso de água $(E / A)$ em milho (C4), arroz (C3) e trigo (C3). Nas três espécies, a curva de resposta de $A$ em função da irradiância $(I)$, apresentou a forma de uma hipérbole retangular, porém em milho não houve saturação lumínica. A resposta de $g$ em relação a $I$ apresentou a mesma forma, respondendo $E$ linearmente, nas três espécies. Em relação à variação de $g$, a curva de resposta de $A$ também mostrou a forma de hipérbole retangular, enquanto $E$ respondeu linearmente. Devido à resposta diferencial de $A$ e de $E$, tanto em função de $I$ como de $g$, a razão $E / A$ aumentou com o aumento de $I$. As espécies C3 (arroz e trigo) revelaram valores maiores de $E / A$ que a $C 4$ (milho), em todos os níveis de $I$ e valores de $g$, indicando melhor adaptabilidade da $C 4$ na limitação de abertura estomática.
\end{abstract}

Termos de indexação: arroz, Oryza sativa L., milho, Zea mays L., trigo, Triticum aestivum L., fotossíntese, transpiração.

\section{ABSTRACT \\ GAS EXCHANGES AND STOMATAL CONDUCTANCE ON THREE GRAMINEOUS SPECIES}

Under natural condition and without water deficit, assimilation of $\mathrm{CO}_{2}(A)$ and transpiration $(E)$ rates, stomatal conductance $(g)$ and photosyntetic efficiency of water use $(E / A)$, were monitored daily on maize (C4), rice (C3) and wheat (C3). In all species, the shape of response curves of $A$ in function of irradiance $(I)$, was a retangular hyperbole. However, luminic saturation was not observed in maize. Stomatal conductance response curve in function of $I$ was also a retangular hyperbole, while $E$ was linear in all species. Due to differential response of $A$ and $E$, as a

( $\left.{ }^{1}\right)$ Trabalho parcialmente financiado pelo CNPq. Recebido para publicação em 23 de setembro e aceito em 30 de novembro de 1994.

(2) Seção de Fisiologia, Instituto Agronômico, Caixa Postal 28, 13001-970 Campinas (SP).

$\left({ }^{3}\right)$ Com bolsa do CNPq. 
function of $I$ as well as $g$, the ratio $E / A$ was increased with the increase of $I$. The C3 species (rice and wheat) showed higher values of $E / A$ than the $C 4$ specie (maize), in all levels of $I$ and $g$, showing the better $C 4$ adaptation when stomatal limitation aperture occurs.

Index terms: rice, Oryza sativa L., maize, Zea mays L., wheat, Triticum aestivum L., photosynthesis, transpiration.

\section{INTRODUÇÃO}

$\mathrm{O}$ acesso de $\mathrm{CO}_{2}$ atmosférico às células fotossintéticas do mesofilo ocorre através da abertura estomática. Por essa mesma via, entretanto, ocorre perda de água no sentido da superfície interna hidratada da folha para a atmosfera. Como a superfície interna da folha é completamente úmida, sendo o gradiente de vapor de água entre a folha e o ar, na maioria dos casos, consideravelmente maior que o gradiente de $\mathrm{CO}_{2}$, em geral, o fluxo de água é centenas de vezes maior que o de $\mathrm{CO}_{2}$ (Farquhar \& Raschke, 1978). Além disso, devido a diferenças nas constantes de difusão de $\mathrm{CO}_{2}$ e de vapor de água no ar, as variações das condutâncias $(g)$ de cada um desses gases também afetam a transpiração $(E)$ e a fotossíntese $(A)$ em diferentes graus (Cowan \& Troughton, 1971; Burrows \& Milthorpe, 1976; Raschke, 1979).

Os fluxos de gases variam com as condições ambientais, sendo dependentes das interações entre as células do mesofilo e dos estômatos (Burrows \& Milthorpe, 1976; Raschke, 1979). O movimento estomático é o meio mais rápido de que a planta dispõe para ajustar-se às variações ambientais a que os órgãos fotossintéticos são submetidos (Passioura, 1982). Condições propícias à fixação de carbono favorecem a abertura do estômato, enquanto condições propícias à perda de água favorecem-lhe o fechamento. A regulação da abertura estomática ocorre por mecanismos complexos (Raschke, 1979), parecendo agir, no entanto, no sentido de minimizar as perdas de água, limitando de forma menos intensa o influxo de $\mathrm{CO}_{2}$ (Cowan \& Troughton, 1971), ou seja, os estômatos parecem funcionar no sentido de otimizar a relação E/A (Cowan, 1982; Farquhar \& Sharkey, 1982). As diversas espécies apresentam diferentes estratégias de adaptação para minimizar a relação $E / A$.
Estudos detalhados das respostas e regulação da abertura estomática, em função da variação do estádio fisiológico, da espécie e de cada fator ambiental, têm sido feitos com sucesso em laboratório (Burrows \& Milthorpe, 1976; Raschke, 1979).Tais estudos têm indicado que a luz, a disponibilidade hídrica no solo e a umidade relativa são os principais fatores ambientais que afetam o comportamento estomático. Sob condições naturais, devido à variação de fatores ambientais, simultaneamente, a avaliação dos mecanismos de regulação da abertura estomática e das trocas gasosas é mais complexa (Schulze \& Hall, 1982). Estudos desta natureza, porém, são fundamentais para compreender os processos adaptativos das espécies agrícolas, quando exploradas comercialmente.

Neste trabalho, analisou-se, sob condiçóes naturais e sem deficiência hídrica, o comportamento da condutância estomática e das taxas de transpiração e de assimilação de $\mathrm{CO}_{2}$ e a eficiência fotossintética de uso de água em arroz, milho e trigo.

\section{MATERIAL E MÉTODOS}

Foram utilizados os cultivares Pioneer 3072 de milho, IAC-165 de arroz e IAC-60 de trigo. Cada espécie foi cultivada em cinco tanques de alvenaria contendo terra, com a seguinte composição: $\mathrm{pH} 6,1$, $3,5 \%$ de matéria orgânica, $120 \mu \mathrm{g}$ de $\mathrm{P} / \mathrm{cm}^{3}$ e 0,57 , 4,2 e $1,9 \mathrm{cmol} / \mathrm{kg}$ de terra, respectivamente, de $\mathrm{K}$, $\mathrm{Ca}$ e $\mathrm{Mg}$. Cada tanque media 4,0 m de comprimento por $0,5 \mathrm{~m}$ de largura por $0,6 \mathrm{~m}$ de profundidade, estando dispostos paralelamente e distantes uns dos outros 0,40 m, sob telado, no Centro Experimental de Campinas (IAC).

Cada espécie foi cultivada no período do ano recomendado para sua produção agrícola econômica. O milho foi semeado em 13/11/90, em linha 
única no centro do tanque, mantendo-se 24 plantas por tanque; o arroz, em 10/12/91, também em linha única, três sementes a cada $7 \mathrm{~cm}$, obtendo-se, após desbaste, uma populaçao de 57 touceiras por tanque, e o trigo, em 18/5/92, em duas linhas espaçadas de $0,3 \mathrm{~m}$, obtendo-se, após a emergência, uma população de 600 plantas por tanque.

Durante todo o ciclo de desenvolvimento das plantas, a umidade da terra foi mantida ao redor de $33 \% \mathrm{massa} / \mathrm{massa}$ (equivalente ao potencial hídrico do solo ao redor de $-0,01 \mathrm{MPa}$ ), de forma a não ocorrer deficiência hídrica para as plantas, devido a solo seco.

As medidas de trocas gasosas, nas três espécies, foram feitas após a floração, sob condições naturais, entre as 7 e as $13 \mathrm{~h}$. Em milho, as medidas foram feitas entre 21 e 24 de janeiro de 1991; em arroz, entre 5 e 8 de março de 1992 e em trigo, entre 4 e 8 de agosto de 1992. Para trigo e arroz, utilizaram-se as folhas bandeiras e, para o milho, a folha inserida na base da espiga. Essas folhas são as mais importantes no fornecimento de substrato fotossintetizado, durante o período de enchimento dos grãos (Machado et al., 1992, 1993, 1994a).

A densidade de fluxo de fótons fotossinteticamente ativos, a taxa de assimilação de $\mathrm{CO}_{2}$, a taxa de transpiração, a condutância estomática e a temperatura da folha foram medidas, simultaneamente, com um sistema portátil de medidas de fotossíntese (Li-6200, Licor, Inc.). Para o trigo e o arroz, utilizou-se a câmara de $0,25 \mathrm{~L}$ e, para o milho, a de 4,0 L. As medidas foram feitas em intervalos de aproximadamente $30 \mathrm{~min}$, com cinco a seis repetições. Detalhes dos tratos culturais e das técnicas de medidas encontram-se em Machado et al. (1992, 1993 e 1994a).

As medidas de temperaturas com termômetros de bulbo úmido e de bulbo seco, para a estimativa da pressão de vapor no ar, foram feitas em um posto meteorológico localizado a $200 \mathrm{~m}$ do local dos experimentos. O déficit de pressão (DP) de saturação entre a folha e o ar foi estimado por:

$$
\text { DPVar-folha = PVar - PVfolha }
$$

onde:

PVfolha é a pressão de vapor na folha, considerando $100 \%$ de umidade na cavidade subestomática (Farquhar \& Raschke, 1978) a dada temperatura da folha, e PVar é a pressão de vapor no ar.

\section{RESULTADOS E DISCUSSÃO}

As medidas de trocas gasosas foram efetuadas em dias claros, sem nuvens e com irradiância variando entre $200 \mu \mathrm{mol} \mathrm{m} \mathrm{m}^{-2} \mathrm{~s}^{-1}$, às $7 \mathrm{~h}$, até 2.500 , às $13 \mathrm{~h}$, para as espécies milho e arroz. Já para o trigo, a irradiância máxima atingida foi 1.600 $\mu \mathrm{mol} \mathrm{m}^{-2} \mathrm{~s}^{-1}$ ao redor das $13 \mathrm{~h}$. As temperaturas da folha e do ar e o déficit de pressão de vapor entre o ar e a folha para os dias típicos de medidas das trocas gasosas são apresentados no quadro 1 .

As respostas da taxa de assimilação de $\mathrm{CO}_{2}(A)$, da taxa de transpiração $(E)$ e da condutância estomática $(g)$, em função da densidade de fluxo de fótons fotossinteticamente ativos (irradiância) (I) em milho, arroz e trigo, encontram-se na figura 1. Nas três espécies, as curvas de resposta de $A$ apresentaram a forma característica de uma hipérbole retangular, porém com variações quanto ao valor máximo de $A$ (Amáx) e o ponto de saturação em relação a $I$. Esses modelos de respostas estão de acordo com outros estudos, relatados na literatura para essas mesmas espécies (Tanaka et al., 1966; Beadle et al., 1985; Dwyer \& Stewart, 1986). Amáx foi de 42,27 e $19 \mu \mathrm{mol}$ de $\mathrm{CO}_{2} \mathrm{~m}^{-2} \mathrm{~s}^{-1}$, respectivamente, para milho, arroz e trigo. A fotossíntese não apresentou saturação lumínica em milho, à semelhança do que ocorre entre as espécies C4. Arroz e trigo, porém, espécies C3, apresentaram saturação lumínica, respectivamente, a 1.100 e 800 $\mu \mathrm{mol}$ de fótons $\mathrm{m}^{-2} \mathrm{~s}^{-1}$ (Figura $1 \mathrm{~A}, \mathrm{~B}$ e C). Embora as temperaturas da folha e do ar e o DPV entre a folha e o ar tenham variado significativamente durante os períodos de medidas (Quadro 1), a curva de fotossíntese, sob condições naturais e sem deficiência hídrica, foi determinada, principalmente, pela variação de $I$, conforme também observaram Marshall \& Biscoe (1980). 
As curvas de respostas de $g$ em relação a $I$, nas três espécies, também apresentaram a forma de uma hipérbole retangular, semelhante ao comportamento de $A$ em relação a $I$ (Figura $1 \mathrm{D}, \mathrm{E}$ e F). Os valores máximos de g (gmáx) ocorreram, respectivamente, para trigo, arroz e milho, em irradiâncias ao redor de 450, 600 e $1.500 \mu \mathrm{mol} \mathrm{m} \mathrm{m}^{-2}$ $\mathrm{s}^{-1}$, mostrando que as duas espécies C3 (arroz e trigo) foram mais responsivas em relação a $I$, sob condiçōes naturais. Padrões semelhantes de respostas de $g$ ao incremento de $I$ já foram observados para outras espécies C3 e C4 (Downes, 1971 a; Turner \& Begg, 1973; Raschke, 1979; Hutmacher \& Krieg, 1983; Ceulemans et al.,1989). A irradiância para que gmáx seja atingido varia marcadamente (100 a $2.000 \mu \mathrm{mol} \mathrm{m}{ }^{-2} \mathrm{~s}^{-1}$ ), dependendo da espécie, da idade da folha e do pré-condicionamento das plantas (Turner \& Begg, 1973; Schulze \& Hall, 1982; Dai et al.,1992). Há indicações de que as espécies $\mathrm{C} 4$ requerem maior nível de fluxo de fótons que as espécies C3, para atingir a abertura máxima dos estômatos (Burrows \& Milthorpe, 1976; Schulze \& Hall, 1982; Dai et al.,1992), à semelhança do que ocorreu neste trabalho. Turner \& Begg (1973) observaram que a condutância em milho foi duas vezes menor que em tabaco, à mesma irradiância. Outros fatores, como temperatura, DPV e concentração interna de $\mathrm{CO}_{2}$ também afetam a abertura estomática. No caso presente, entretanto, o comportamento de $g$ deve representar a resultante da variação de todos esses fatores em conjunto (Quadro 1).

A transpiração pode ser estimada a partir da relação $E=g\left(w_{i}-w_{a}\right)$, onde $w_{i}-w_{a}$ representa a diferença entre a fração molar de vapor de água no espaço interno $\left(w_{i}\right)$ e na atmosfera $\left(w_{a}\right)$ (Cowan, 1982). Conseqüentemente, o aumento de $E$ em função de $I$ era esperado (Figura $1 \mathrm{G}, \mathrm{H}$ e I), visto que $g$ também aumentou com $I$ (Figura 1D, E e F). Entretanto, nas três espécies, $E$ aumentou praticamente de forma linear em função de $I$, enquanto $g$ aumentou de forma hiperbólica (Figura 1D, E e F). Quando $I$ aumentou, as temperaturas do ar e da folha e o DPV entre a folha e o ar também aumentaram, proporcionando maior demanda hídrica (Quadro 1). Assim, apesar de $g$ ter-se mantido constante em valores de $I$ acima da saturação (Fi- gura 1D, E e F), a diferença $\left(w_{i}-w_{a}\right)$ aumentou, justificando a relação linear entre $E$ e $I$. Como esses fatores ambientais variaram simultaneamente, é difícil isolar o mais importante, apesar de o efeito de cada um deles estar estabelecido experimentalmente em condições de laboratório (Burrows \& Milthorpe, 1976; Raschke, 1979). Dai et al. (1992) observaram que o aumento de DPV provocou queda em $g$ e em $A$, porém $E$ aumentou.

Comparativamente, o arroz apresentou os maiores valores de $E$, seguido pelo trigo e com valores bem menores, pelo milho (Figura $1 \mathrm{G}, \mathrm{H}$ e I). A maior sensibilidade dos estômatos das espécies $\mathrm{C} 3$ (trigo e arroz) em relação a $I$, combinada com os maiores valores de $g$, implicaram maior perda de água por unidade de área foliar que na espécie $\mathrm{C} 4$ (milho), especialmente nas primeiras horas da manhã, quando os valores de $g$ já atingiram seu valor máximo. Dai et al. (1992) também observaram que os maiores valores de $E$ em espécies C3 (mamona e tabaco) em relação ao milho (C4), foram devidos aos maiores valores de $\mathrm{g}$. Contudo, o maior valor de $A$ em milho, em relação ao trigo e ao arroz, é devido às características anatômicas e metabólicas das espécies C4 (Black, 1985).

A figura 2 mostra respostas de $E$ e de $A$ em função da condutância estomática $(g)$. A variação da abertura estomática afetou a transpiração e a fotossíntese em intensidades diferentes, conforme também observaram Cowan \& Troughton (1971). Embora o aumento de $E$ exerça um efeito refrigerante retroativo (diminui a pressão de vapor na folha), a relação $E$ em função de $g$ é muito próxima da linearidade, devido ao aumento da demanda hídrica, como foi discutido.

A resposta de $A$ em função de $g$, por sua vez, apresentou a forma de uma hipérbole retangular, onde $A$ se apresentou saturado em relação a $g$ em valores acima de $0,7,0,9$ e $0,7 \mathrm{~mol} \mathrm{~m}^{-2} \mathrm{~s}^{-1}$, respectivamente, para milho, arroz e trigo (Figura 2D, E e F). A ausência de resposta de $A$ ao incremento de $g$, acima dos valores de saturação, indica que outros mecanismos, além de abertura estomática, estão controlando a assimilação de $\mathrm{CO}_{2}$ (Hutmacher \& Krieg, 1983). 
Quadro 1. Temperaturas da folha, do ar e déficit de pressão de vapor entre a folha e o ar (DPV)

\begin{tabular}{|c|c|c|c|c|c|c|}
\hline \multirow{2}{*}{ Variável } & \multicolumn{2}{|c|}{ Temperatura da folha } & \multicolumn{2}{|c|}{ Temperatura do ar } & \multicolumn{2}{|c|}{ DPV } \\
\hline & $7 \mathrm{~h}$ & $13 \mathrm{~h}$ & $7 \mathrm{~h}$ & $13 \mathrm{~h}$ & $7 \mathrm{~h}$ & $13 \mathrm{~h}$ \\
\hline & $\longrightarrow$ & - & $\longrightarrow$ & - & $\longrightarrow$ & 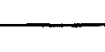 \\
\hline Milho & 22,3 & 34,0 & 21,3 & 31,2 & $-0,3$ & $-1,33$ \\
\hline Arroz & 22,0 & 34,0 & 22,0 & 31,5 & $-0,8$ & $-1,40$ \\
\hline Trigo & 17,5 & 28,1 & 13,6 & 26,6 & $-0,08$ & $-0,78$ \\
\hline
\end{tabular}

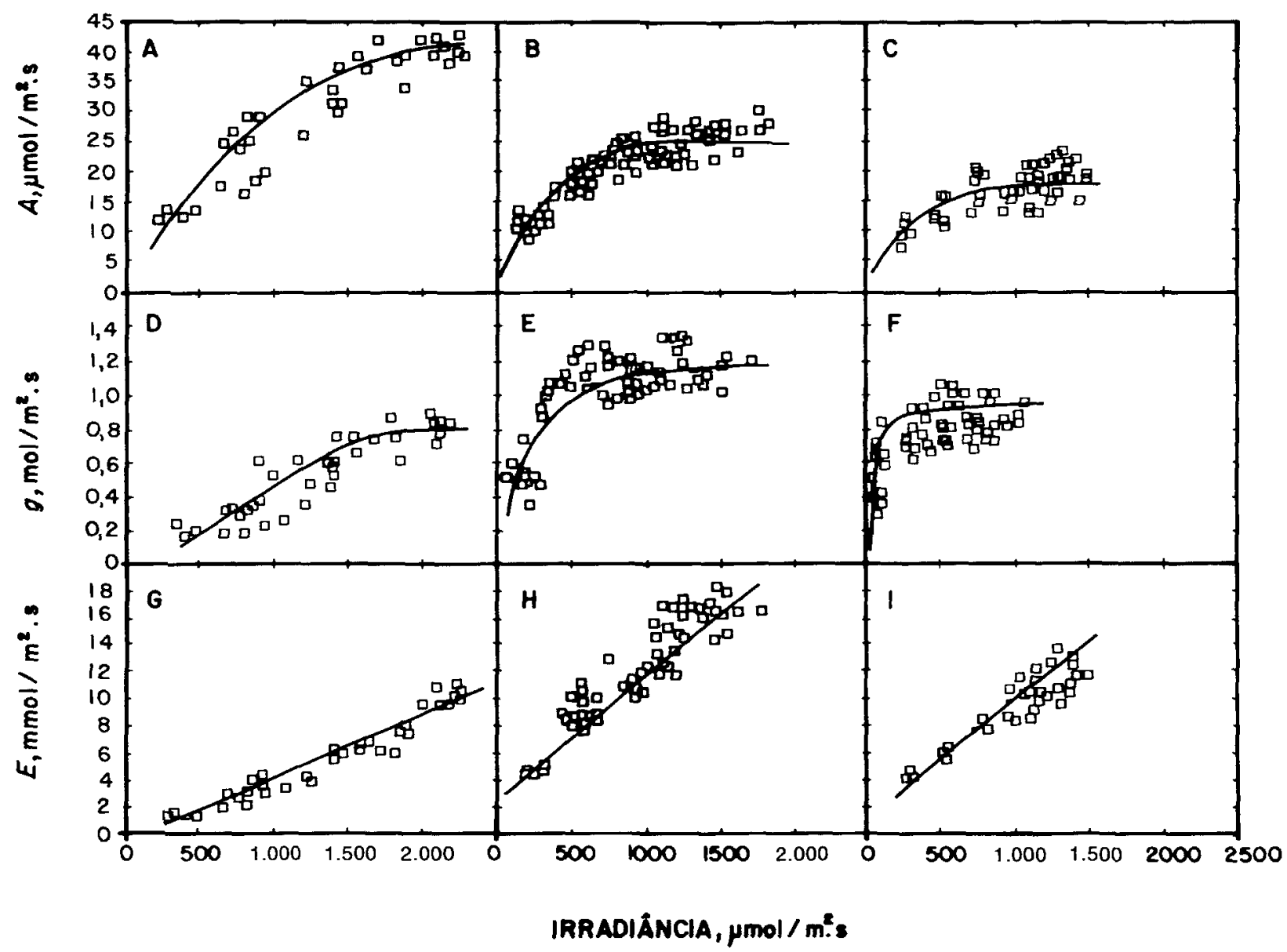

Figura 1. Respostas da taxa de fotossíntese $(A)$, da condutância estomática $(g)$ e da taxa de transpiração (E) em função da irradiância, em milho (A, D e G), arroz (B, E e H) e trigo (C, F e I), sob condiçōes naturais. 


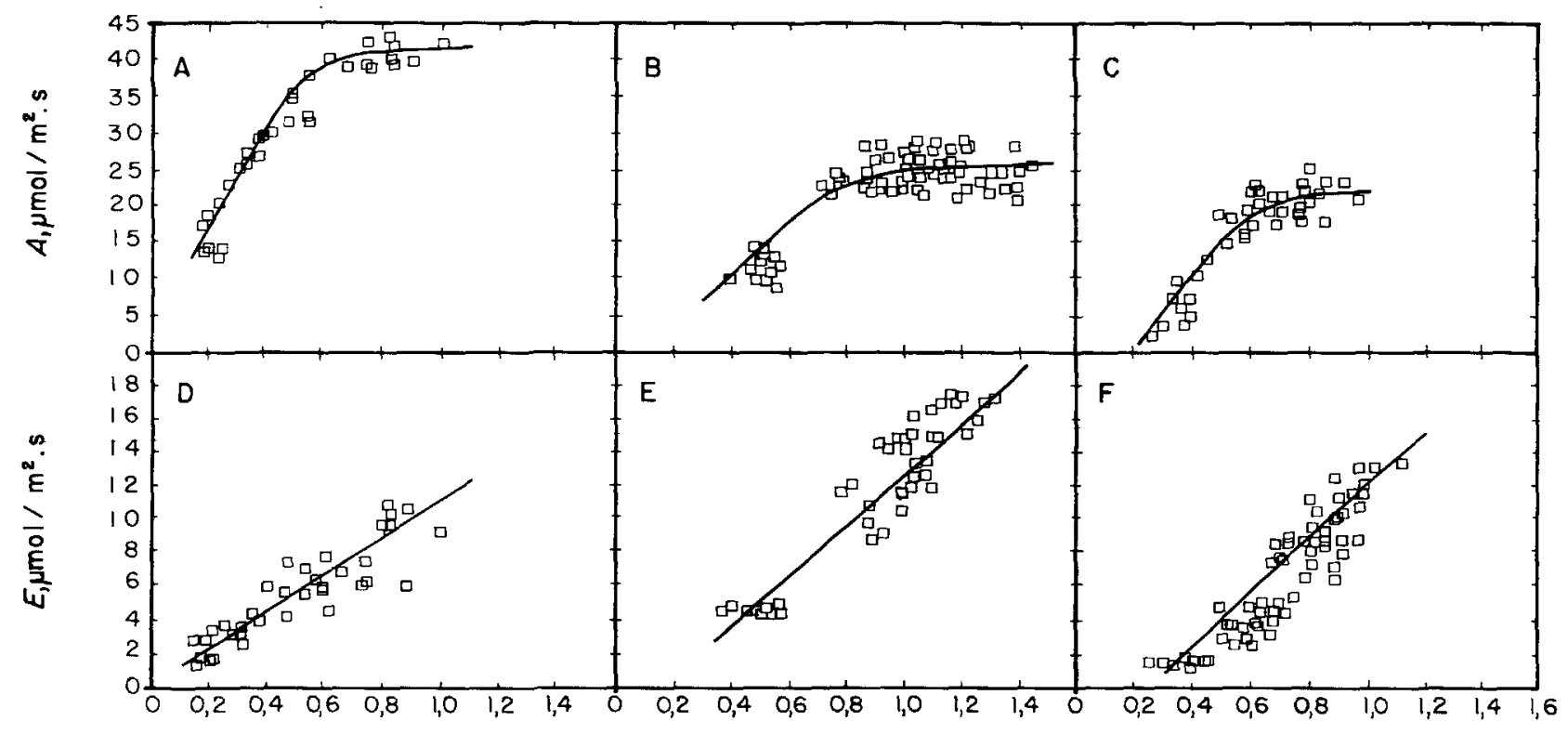

CONDUTÂNCIA, $\mathrm{mol} / \mathrm{m}^{2} . \mathrm{s}$

Figura 2. Respostas das taxas de fotossintese $(A)$ e de transpiração $(E)$ à variação da condutância estomática (g) em milho (A e D), arroz (B e E) e trigo (C e F), sob condições naturais.

A taxa de assimilação de $\mathrm{CO}_{2}$ pode ser estimada pela relação $A=g\left(c_{i}-c_{a}\right)$, onde $\left(c_{i}-c_{a}\right)$ representa a diferença da concentração molar de $\mathrm{CO}_{2}$ entre o espaço intercelular $\left(c_{i}\right)$ e a atmosfera $\left(c_{a}\right)$. Quando $I$ e as demais condições atmosféricas se mantêm constantes, $A$ e a concentração interna de $\mathrm{CO}_{2}$ tendem a manter-se constantes (Farquhar \& Sharkey, 1982). Quando $I$ aumenta, $A$ também aumenta, até certos limites característicos de cada espécie e dependentes do tipo de metabolismo fotossintético $\mathrm{C} 3$ ou C4 (Figura 1A, B e C). O aumento de $A$ provoca queda na concentração interna de $\mathrm{CO}_{2}$, exercendo forte efeito retroativo negativo, podendo, conseqüentemente, causar queda em $A$. A queda na concentração interna de $\mathrm{CO}_{2}$, porém, estimula maior abertura estomática, permitindo maior penetração de $\mathrm{CO}_{2}$ para a cavidade subestomática (Raschke, 1979; Dai et al., 1992). O mecanismo de abertura estomática em resposta à concentração interna de $\mathrm{CO}_{2}$ parece agir também em conjunto com outros estímulos, como intensidade e qualidade da radiação solar (Raschke, 1979; Farquhar \& Sharkey, 1982).
Autores demonstraram o aumento de $A$ e da concentração interna de $\mathrm{CO}_{2}$ com o aumento de $g$, como Raschke (1979); Farquhar \& Sharkey (1982); Cornish et al.(1991); Dai et al.(1992). Para baixa concentração interna de $\mathrm{CO}_{2}$, a enzima ribulose bisfosfato $\left(\mathrm{RuP}_{2}\right)$ carboxilase-oxigenase (rubisco) fica saturada com respeito ao substrato $\mathrm{RuP}_{2}$. O aumento da concentração interna de $\mathrm{CO}_{2}$ provoca aumento linear de $A$ até a fotossíntese atingir o valor máximo. Incrementos na fotossíntese, além desse ponto, não ocorrem devido à saturação de $\mathrm{CO}_{2}$ (Cowan, 1982) e à limitação quanto à capacidade de regeneração de $\mathrm{RuP}_{2}$ (Farquhar \& Sharkey, 1982), ou seja, mesmo que ocorra um aumento na concentração interna de $\mathrm{CO}_{2}$, não ocorre aumento de $A$.

$\mathrm{Na}$ figura 2, verifica-se que, na região da curva onde há ausência de resposta de $A$ em função de $g$, o aumento na condutância provoca aumentos somente em $E$ e, conseqüentemente, há queda na eficiência fotossintética do uso de água. A figura 3 (A, B e C) mostrou essa relação entre $A$ e $E$, ou seja, $E$ continua a crescer mesmo após $A$ ter atingido 
seu valor máximo. Comportamento semelhante foi descrito por outros autores, tanto em espécies C3 como em C4 (Cowan \& Troughton, 1971; Dubbe et al.,1978; Farquhar et al.,1980; Vu et al.,1986; Machado et al.,1994b). Sob condições controladas, em níveis comparáveis de $g$, Downes (1971b) de-

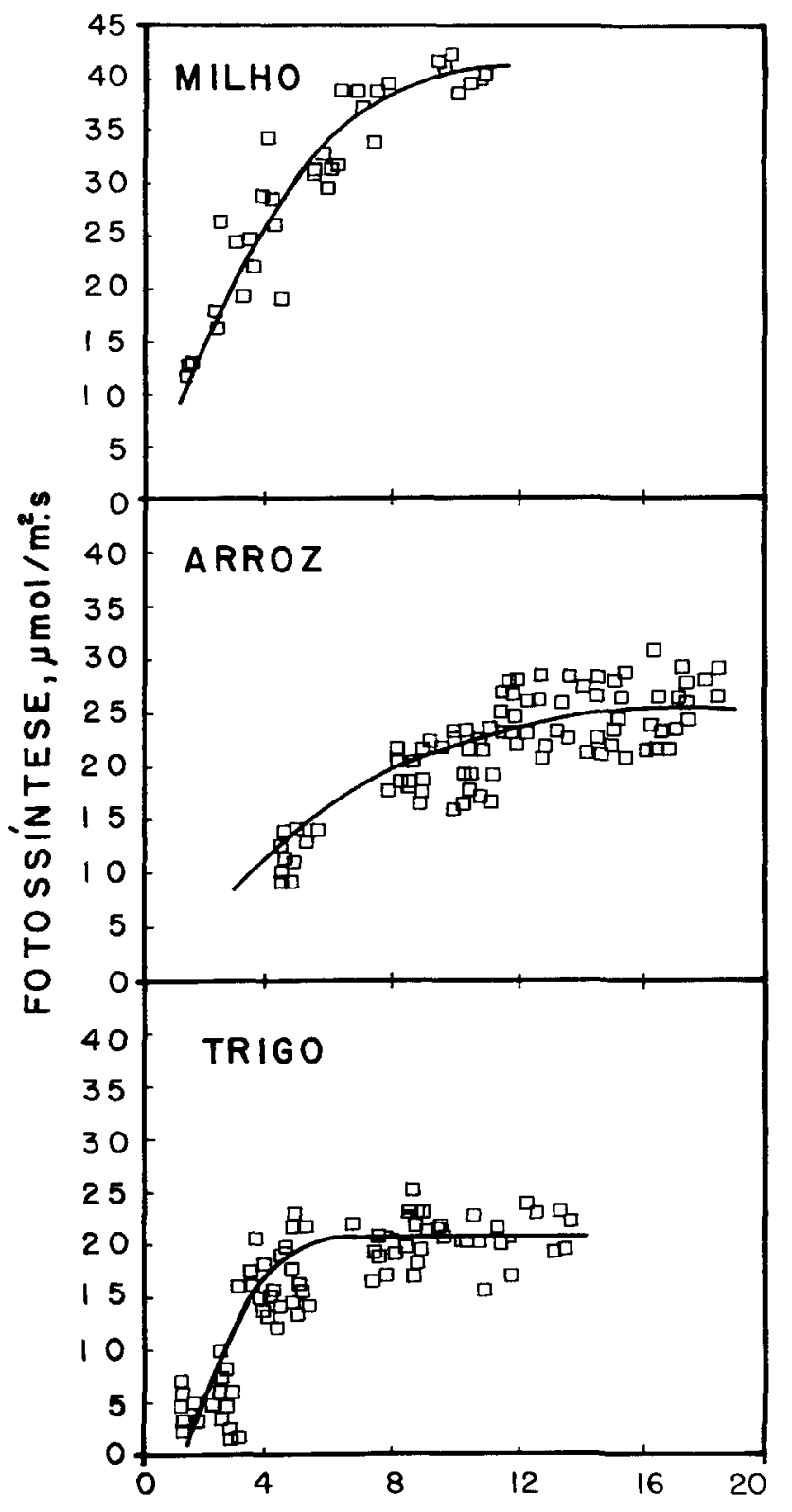

TRANSPIRAÇ Á $0, \mathrm{mmol} / \mathrm{m}^{2} . \mathrm{s}$

Figura 3. Relaçăo entre as taxas de fotossíntese e de transpiração, em milho, arroz e trigo, sob condiçōes naturais. monstrou que a fotossíntese em sorgo (C4) foi maior que em trigo, sugerindo maiores vantagens das espécies $\mathrm{C} 4$ para condições de estresse. A partir da figura 3 , verifica-se que a relação $A m a ́ x / E$ foi de $5,3.10^{-3} \mathrm{mmol}$ de $\mathrm{CO}_{2} / \mathrm{mmol}$ de $\mathrm{H}_{2} \mathrm{O}$ para o milho, $4,0.10^{-3}$ para o trigo e $2,8.10^{-3}$ para o arroz.

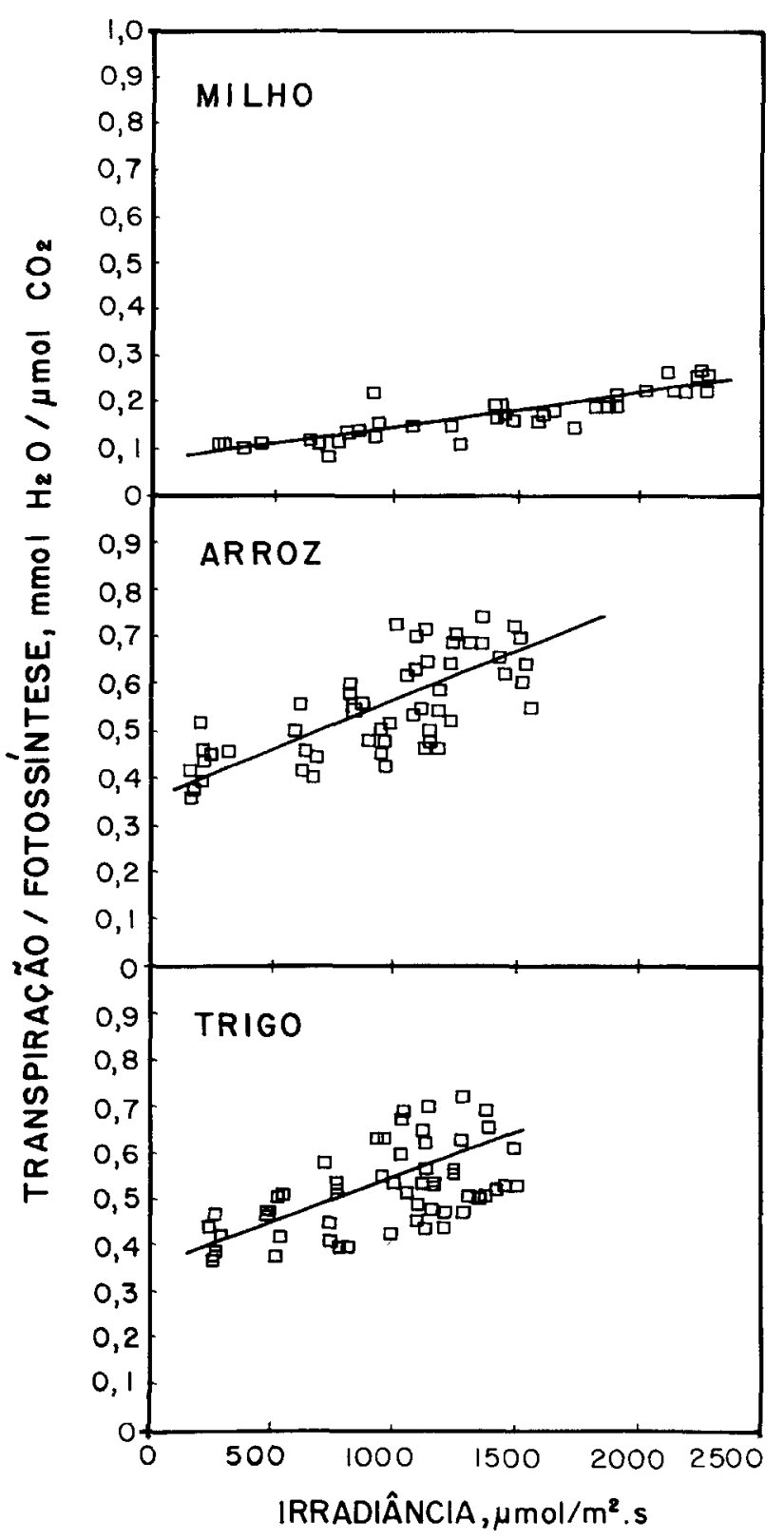

Figura 4. Variação da eficiência fotossintética do uso da água (razão transpiração/fotossintese) em função da irradiância, em milho, arroz e trigo, sob condições naturais. 
A figura 4 mostrou que a relação $E / A$ aumentou em função da variação de $I$ em milho, arroz e trigo. Tal comportamento é explicado pelo fato de que $E$ cresceu linearmente, enquanto $A$ aumentou de forma hiperbólica, tanto com o aumento de $I$ como de $g$ (Figura 2). Cowan \& Troughton (1971) também observaram que a relação $E / A$ diminuiu com a elevação de $g$ em função do comportamento diferencial entre $E$ e $A$. Outro fato que provocou a queda da relação $E / A$ foi o aumento de DPV com o aumento de $I$.

Tanto a relação $E / A$, como seu aumento em resposta a $I$, foram maiores nas espécies $\mathrm{C} 3$ que no milho, para qualquer nível de $I$. Downes (1971b) sugeriu que a eficiência fotossintética do uso de água nas espécies $\mathrm{C} 4$ é uma das causas de sua vantagem adaptativa sobre C3, quando há limitação da abertura estomática.

\section{REFERÊNCIAS BIBLIOGRÁFICAS}

BEADLE, C.L.; LONG, S.P.; IMBAMBA, S.K.; HALL, D.O. \& OLEMBO, R.J. Photosynthesis in relation to plant production in terrestrial environments. 1 .ed. Oxford, Tycooly Publishing, 1985. 156p.

BLACK, C.C. Efficiency of carbon assimilation. In: NEYRA, C.A., ed. Biochemical basis of plant breeding: carbon metabolism. Boca Raton, C.R.C. Press, 1985. v. 1, p. $73-88$.

BURROWS, F.J. \& MILTHORPE, F.L. Stomatal conductance in the control of gas exchange. In: KOZLOWSKI, T.T., ed. Water deficits and plant growth. New York, Academic Press, 1976. v. 4, p. $103-152$.

CEULEMANS, R.; HINCKLEY, T.M. \& IMPENS, I. Stomatal response of hybrid poplar to incident light, sudden darkening and leaf excision. Physiologia Plantarum, Copenhagen, 75(2):174-182, 1989.

CORNISH, K.; RADIN, J.W.; TURCOTTE, E.L.; LU, Z. \& ZEIGER, E. Enhanced photosynthesis and stomatal condutance of pima cotton (Gossypium barbadense L.) bred for increased yield. Plant Physiology, Lancaster, 97(2):484-489, 1991.
COWAN, I.R. Regulation of water use in relation to carbon gain in higher plants. In: LANGE, O.L.; NOBEL, P.S.; OSMOND, C.B. \& ZIEGLER, H., eds. Physiological plant ecology. II. Water relations and carbon assimilation. Berlin, Springer-Verlag, 1982. v. 12B, p. 589-613. (Encyclopedia of plant physiology)

COWAN, I.R. \& TROUGHTON, J.H. The relative role of stomata in transpiration and assimilation. Planta, Berlin, 97(4):325-336, 1971.

DAI, Z.; EDWARD, G.E. \& KU, M.S.B. Control of photosynthesis and stomatal condutance in Ricinus communis $\mathrm{L}$. (Castor bean) by leaf to air vapor pressure deficit. Plant Physiology, Lancaster, 99(4):14261434, 1992.

DOWNES, R.W. Relationship between evolutionary adaptation and gas exchange characteristics of diverse sorghum taxa. Australian Journal of Biological Science, Melbourne, 24(4):843-852, 1971a.

DOWNES, R.W. Adaptation of sorghum plants to light intensity: its effect on gas exchange in response to changes in light, temperature, and $\mathrm{CO}_{2}$. In: $\mathrm{HATCH}$, M.D.; OSMOND, C.G. \& SLATYER, R.O., eds. Photosynthesis and photorespiration. New York, Wiley Interscience, 1971b. p. 57-62.

DUBBE, D.R.; FARQUHAR, G.D. \& RASCHKE, K. Effect of abscisic acid on the gain of the feedback loop involving carbon dioxide and stomata. Plant Physiology, Lancaster, 62(3):413-417, 1978.

DWYER, M. \& STEWART, D.W. Effect of leaf age and position on net photosynthetic rates in maize (Zea mays L.). Agricultural and Forest Meteorology, Amsterdam, 37(1):29-46, 1986.

FARQUHAR, G.D. \& RASCHKE,K. On the resistance to transpiration of the sites of evaporation within the leaf. Plant Physiology, Lancaster, 60(6): 1000-1005, 1978.

FARQUHAR, G.D.; SCHULZE, E.D. \& KÜPPERS, M. Responses to humidity by stomata of Nicotina glauca L. and Corylus avellana L. are consistent with the optimization of carbon dioxide uptake with respect to water loss. Australian Journal of Plant Physiology, Melbourne, 7(3):315-327, 1980.

FARQUHAR, G.D. \& SHARKEY, T.D. Stomatal condutance and photosynthesis. Annual Review of Plant Physiology, Boca Raton, 33:317-345, 1982. 
HUTMACHER, R.B \& KRIEG, D.R. Photosynthetic rate control in cotton. Stomatal and nostomatal factors. Plant Physiology, Lancaster, 73(3):658-661, 1983.

MACHADO, E.C.; LAGÔA, A.M.M.A. \& TICELLI, M. Relações fonte-dreno em trigo submetido à deficiência hídrica no estádio reprodutivo. Revista Brasileira de Fisiologia Vegetal, São Carlos, 5(2):145-150, 1993.

MACHADO, E.C.; LAGÔA, A.M.M.A.; TICELLI, M.; AZZINI, L.E. \& TISSELLI $\mathrm{F}^{\circ}$, O. Fotossíntese $\mathrm{e}$ crescimento de panículas em arroz de sequeiro (Oryza sativa $\mathrm{L}$. c v. IAC-165). Revista Brasileira de Botânica, São Paulo, 17(1):37-43, 1994a.

MACHADO, E.C.; QUAGGIO, J.A.; LAGÔA, A.M.M.A.; TICELLI, M. \& FURLANI, P.R. Trocas gasosas e relações hídricas em laranjeiras com clorose variegada dos citros. Revista Brasileira de Fisiologia Vegetal, São Carlos, 6(1):53-57, 1994b.

MACHADO, E.C.; SILVEIRA, J.A.G. da; VITORELLO, V.A. \& RODRIGUES, J.L.M. Fotossíntese, remobilização de reservas e crescimento de grãos em dois híbridos de milho sob deficiência hídrica na fase de enchimento dos grãos. Bragantia, Campinas, 51(2): $151-159,1992$.

MARSHALL, B. \& BISCOE, P.V. A model for C3 leaves descriting the dependence of net photosynthesis on irradiance: I. Derivation. Journal of Experimental Botany, Oxford, 31(120):29-39, 1980.
PASSIOURA, J.B. Water in the soil-plant-atmosphere continuum. In: LANGE, O.L.; NOBEL, P.S.; OSMOND, C.B. \& ZIEGLER,H., eds. Physiological plant ecology. II. Water relations and carbon assimilation. Berlin, Springer-Verlag, 1982. v. 12B, p. 5-33. (Encyclopedia of plant physiology)

RASCHKE, K. Movements using turgor mechanisms. In: HAUPT, W. \& FEINLEIB, M.E., eds. Physiology of movements. Berlin, Springer-Verlag, 1979. v. 7 , p.383-441. (Encyclopedia of piant physiology)

SCHULZE, E.D. \& HALL, A.E. Stomatal responses, water loss and $\mathrm{CO}_{2}$ assimilation rates of plants in contrasting environments. LANGE, O.L.; NOBEL, P.S.; OSMOND, C.B. \& ZIEGLER, H., eds. Physiological plant ecology. II. Water relations and carbon assimilation. Berlin, Springer-Verlag, 1982. v. 12B, p.181-230. (Encyclopedia of plant physiology)

TANAKA, A.; KAWANO, K. \& YAMAGUCHI, J. Photosynthesis, respiration, and plant type of the tropical rice plant. Los Baños, the International Rice Research Institute, 1966. 46p. Technical Bulletin, 7.

TURNER, N.C. \& BEGG, J.E. Stomatal behavior and water status of maize, sorghum, and tobacco under field conditions. I. At high soil water potential. Plant Physiology, Lancaster, 51(1):31-36, 1973.

VU, J.C.V.; YELENOSKY, G. \& BAUSHER, M.G. $\mathrm{CO}_{2}$ exchange rate, stomatal condutance, and transpiration in attached leaves of 'Valência' orange. HortScience, Alexandria, 21(1):143-144, 1986. 